ANOUCHKA VASAK

ORCID: 0000-0002-7020-0863

Université de Poitiers

anouchka.vasak-chauvet@wanadoo.fr

\title{
THÉÂTRE ET THÉRAPIE DE LA MALADIE MENTALE (FRANCE, 1790-1815)
}

On a parlé de la " théâtromanie » qui envahit la France dans le dernier quart du XVIII ${ }^{\mathrm{e}}$ siècle. On connaît mieux désormais le " théâtre patriotique » qui fleurit sous la Révolution grâce aux travaux de Philippe Bourdin ${ }^{1}$. Je voudrais pour ma part m'arrêter sur les relations singulières qui lient le théâtre et la thérapie de la maladie mentale pendant la Révolution et l'Empire : elles ne relèvent pas du théâtre institué, et il est difficile de les situer dans un cadre général. Mais ces expériences soulèvent également des questions politiques. La période se caractérise par un nouveau regard porté sur la maladie mentale, notamment à travers le « traitement moral des aliénés » introduit par Philippe Pinel. Celui qu'on appellera « le bienfaiteur des aliénés », mais surtout certains de ses émules plus ou moins fidèles, ont pu considérer le théâtre comme un moyen thérapeutique. Qu'entendon alors par théâtre?

Le mot « théâtre » désigne un genre (« poésie dramatique ») et un lieu, « Sorte d'échafaud — dit le Dictionnaire de l'Académie de 1762 — sur lequel on représente des tragédies, des comédies, des opéra $\&$ d'autres spectacles, \& où l'on danse des ballets, \&c. ». Je l'emploierai quant à moi au sens propre comme au figuré :

- dans un sens métaphorique : le théâtre, ou plus exactement la mise en scène, est utilisé comme thérapie dans certains cas rapportés par Pinel ;

${ }^{1} \mathrm{Ph}$. Bourdin, Aux origines du théâtre patriotique, CNRS Éditions, Paris 2017. 
— dans son sens propre : je m'arrêterai sur la fameuse expérience théâtrale menée entre 1805 et 1813 par le marquis de Sade à l'hospice de Charenton où il est alors interné, et où il mourra en 1814.

Pourquoi un lien se noue-t-il à ce moment-là de l'histoire entre théâtre et maladie mentale ? Quel sens politique conférer à cette association, en cette période qui verra la thérapie de la maladie mentale se transformer au rythme des changements de pouvoir? Enfin, quelles lectures faisons-nous (ou avons-nous faites), depuis les entours de Mai 68 jusqu'à aujourd'hui, de l'étonnante expérience théâtrale de Sade à Charenton?

Je m'efforcerai de répondre à ces questions en considérant trois moments :

1. Sous la Révolution : le traitement moral des aliénés, en particulier dans les cas produits par le traumatisme révolutionnaire.

2. Sous l'Empire : l'expérience du théâtre de Charenton menée par Sade et soutenue jusqu'à un certain point par le directeur de l'hospice, Coulmier.

3. Aujourd'hui $\left(\mathrm{XX}^{\mathrm{e}}\right.$ - début $\mathrm{XXI}^{\mathrm{e}}$ siècles $)$ : comment relisons-nous l'expérience de Charenton (Marat/Sade de Peter Weiss, 1964) et que révèle dans le théâtre contemporain, de façon plus générale, la représentation de la Révolution ? Quelle valeur sinon thérapeutique, du moins politique, peut-on lui attribuer?

\section{LE THÉÂTRE DANS LE TRAITEMENT MORAL DES ALIÉNÉS}

C'est sous le Directoire, en mai 1797 (Prairial an V) que le gouverneur de l'hôpital de Bicêtre, Jean-Baptiste Pussin, supprime les chaînes aux aliénés. Et c'est nourri de cette pratique que Philippe Pinel, médecin des aliénés de Bicêtre et médecin-chef de la Salpêtrière depuis 1795, met en place le « traitement moral ». Psychologique en réalité, ce traitement a pour principe de faire appel à l'intelligence du patient. L'auteur du Traité médico-philosophique montre en effet qu'il subsiste toujours des traces de raison chez l'aliéné : c'est la notion d'intermittence. Le premier chapitre de cet ouvrage, écrit en 1797, identifie la période révolutionnaire comme favorable à l'observation des aliénés :

L'hospice de Bicêtre, confié à mes soins, à titre de médecin, durant l'an 2 et l'an 3 de la République, m'ouvrit un vaste champ pour poursuivre mes recherches sur la Manie, commencées à Paris depuis quelques années. Quelle époque plus favorable que celle des grands orages de la révolution, toujours propre à donner une activité brûlante aux passions, ou plutôt à produire la manie sous toutes ses formes $^{2}$.

Le nombre des aliénés reçus à Bicêtre ne semble pas avoir augmenté de façon significative entre 1789 et 1793, mais Pinel fait état du recensement effectué

$2 \mathrm{Ph}$. Pinel, Traité médico-philosophique sur l'aliénation mentale, ou La Manie, section II : «Circonstances favorables pour mes recherches sur la Manie », an IX (1800), L'Harmattan, Paris 2006, p. 9. 
en l'an III (1794) dans cet hôpital et observe que sur cent-treize aliénés « sur lesquels [il a] pu obtenir des informations exactes », trente " avaient été réduits à cet état $[\ldots]$ par des événements de la révolution ${ }^{3} »$, soit environ un quart de la population de l'hospice. Dans L'homme qui se prenait pour Napoléon ${ }^{4}$, Laure Murat montre que la Révolution française a tout à la fois généré de nouvelles pathologies mentales, des cas d'aliénation directement liés aux «événements », et jeté les bases du traitement médical propre à traiter ces pathologies. Et si Pinel s'inspire d'expériences thérapeutiques pratiquées ailleurs en Europe (Angleterre, Vienne, Hollande), il y a bien un lien entre la Révolution française et le traitement moral : «Le lien entre la révolution et le traitement moral, écrit Jan Goldstein, fut bien plus que circonstanciel : il détermine la nature même de la thérapie. [...]. La douceur est à la fois posée comme un traitement médical et une exigence politique $»^{5}$.

Autre marque de ce nouveau regard porté sur la folie, sensible dans les ouvrages de Pinel : un mode d'écriture plus littéraire que médical qui fait exister l'individualité des aliénés. Pinel a conscience de l'effet de fiction produit par les récits de cas, qu'il puise, dit-il, dans un « fonds inépuisable d'historiettes »; mais il prend garde à rester sur le terrain médical ${ }^{6}$. Portraits et saynètes, embryons de fables même, se succèdent dans ses récits. Pourtant, le théâtre est plus qu'un mode de restitution plaisant de cas : c'est un moyen thérapeutique, qui produit des « fictions curatives $»^{7}$ (Jean Starobinski).

Cependant, le théâtre entendu comme moyen thérapeutique n'est pas une invention de Pinel. Cette pratique rappelée notamment, mais pas uniquement, par Michel Foucault dans Naissance de la clinique ${ }^{8}$, s'inscrit dans une tradition issue de l'Antiquité. À la fin du XVIII ${ }^{\mathrm{e}}$ siècle elle correspond à un programme sensualiste, fondé sur ce nouveau regard porté sur le sujet promu par Locke ou Condillac. L'" aliéné » pourrait, à la faveur d'une thérapie mettant en œuvre des stimuli extérieurs, recouvrer la santé mentale oblitérée. Philippe Pinel à Paris, mais aussi Vincenzo Chiarugi à Florence, Benjamin Rush à Philadelphie, William Tuk à York sont les artisans de ce renouveau thérapeutique appelé communément

${ }^{3}$ Ibidem, p. 110.

${ }^{4}$ L. Murat, L'homme qui se prenait pour Napoléon. Pour une histoire politique de la folie, Gallimard, Paris 2011.

5 J. Goldstein, Consoler et classifier. L'essor de la psychiatrie française, Cambridge 1987 [première édition] ; Institut Synthélabo, Paris 1997 [réédition].

${ }^{6} \mathrm{Ph}$. Pinel, op. cit., section VI : «Principes du traitement médical des aliénés » ( $\$ 3$ : « La Guérison de la mélancolie souvent très difficile ; variété des moyens à mettre en usage »), pp. 231-232.

7 J. Starobinski, L'encre de la mélancolie, Éditions du Seuil, Paris 2012.

${ }^{8}$ M. Foucault, Naissance de la clinique, Presses universitaires de France, Paris 1963 ; B. Simon, Mind and Madness in Ancient Greece: The Classical Roots of Modern Psychiatry, Cornell University Press, Ithaca 1980. 
« traitement moral $»^{9}$. Si le patient ne peut être convaincu rationnellement de ses erreurs, provoquer en lui des « chocs » émotionnels peut avoir un effet thérapeutique immédiat. Ces chocs sont de trois ordres : physiques - ce sont les douches ou les « bains de surprise », esthétiques - favoriser une émotion à travers l'art ou la musique, psychiques - mise en scène d'un événement qui résout à son insu l'obsession du patient ${ }^{10}$. C'est dans cette dernière catégorie que prend place l'usage du théâtre comme moyen thérapeutique, ainsi que la mise en scène montée à l'insu du patient appelée pious fraud (Mary de Young), fraude pieuse. C'est précisément ce que Pinel, prenant appui sur les traumatismes produits par l'époque révolutionnaire, met en œuvre en certaines occasions.

On peut ainsi s'arrêter sur deux cas particuliers évoqués par Pinel dans son Traité médico-philosophique. Le premier patient a été " guéri », au moins provisoirement, par Pussin ; Pinel raconte la mise en scène dans un chapitre intitulé «Avantage d'ébranler fortement l'imagination d'un aliéné dans certains cas » :

Un jeune homme consterné du renversement du culte catholique en France, et dominé par des préjugés religieux, devint maniaque [...]. Rien n'égale sa sombre misanthropie ; il ne parle que des tourments de l'autre vie, et il pense que pour s'y soustraire, il doit imiter les abstinences et les macérations des anciens anachorètes ; il s'interdit dès lors toute nourriture, et vers le quatrième jour de cette résolution inébranlable, son état de langueur fait craindre pour sa vie.

Rien, rapporte Pinel, ne peut le dissuader de ce jeûne obstiné.

Intervient alors la mise en scène de Pussin, propre selon lui à « contrebalancer » le cours des idées sinistres du « maniaque »:

[...] Le citoyen Pussin se présente le soir à la porte de sa loge, avec un appareil propre à effrayer, l'œil en feu, un ton de voix foudroyant, un groupe de gens pressés autour et armés de fortes chaînes qu'ils agitent avec fracas ; on met un potage auprès de l'aliéné et on lui intime l'ordre le plus précis de le prendre durant la nuit, s'il ne veut pas encourir les traitements les plus cruels ${ }^{11}$.

Cette mise en scène naïve est efficace : le jeune homme, après une nuit d'hésitations, cède et entre dès lors en convalescence...

Un tel expédient peut faire sourire. Mais un autre cas, traité par Pinel luimême, utilise délibérément la théâtralité comme un moyen thérapeutique. C'est celui d'un ouvrier tombé dans une " mélancolie profonde » parce qu'il a laissé « un jour échapp[er] en public quelques réflexions sur le jugement et la condamnation de Louis XVI ». Pour guérir l'aliéné, Pinel imagine, en accord avec trois jeunes médecins, une véritable mise en scène :

${ }^{9}$ C. Raz, « Musique, théâtre et traitement moral : la Casa dei Matti d'Aversa et celle de Palerme », Laboratoire italien 20, 2017, ENS Éditions (<https://journals.openedition.org/laboratoireitalien/1581\#bodyftn2> [consulté le 11.10.2019]).

10 M. de Young, Encyclopedia of Asylum Therapeutics (1750-1950s), McFarland \& Company, Jefferson (NC) 2015, pp. 344-345.

11 Ph. Pinel, op. cit., pp. 50-60. 
Je me concerte donc avec trois jeunes médecins, et je charge du principal rôle celui qui a l'air le plus grave et le plus imposant. Ces commissaires en habit noir et avec tout l'appareil de l'autorité, se rangent autour d'une table et font comparaître le mélancolique.

Est alors prononcée, de façon prétendument officielle, une sentence d'acquittement, qui aura pour effet de libérer l'aliéné de sa mélancolie :

Nous, commissaires, en vertu du plein pouvoir qui nous a été accordé par l'Assemblée nationale, avons procédé, suivant les formes usitées, à l'examen juridique du cit..., et nous reconnaissons n'avoir trouvé en lui que les sentiments du plus pur patriotisme; il est donc acquitté de toute poursuite intentée contre lui, et ordonnons qu'il recouvre sa liberté entière et qu'il soit rendu à sa famille ${ }^{12}$.

Si la guérison s'avérera de courte durée, le malade ayant eu vent du stratagème, ce cas, inspiré par la mise en scène du tribunal révolutionnaire, révèle peut-être une conscience nouvelle, laïque, de la théâtralité du pouvoir. On sait, et on y reviendra, combien certains de nos metteurs en scène contemporains ont su s'emparer de ce dispositif.

D'autres expériences thérapeutiques similaires sont pratiquées en Europe à la même période. Plusieurs cas passionnants sont décrits et analysés par Carmel $\mathrm{Raz}^{13}$, tels celui de la Casa dei Matti d'Aversa près de Naples, ou de l'hôpital de Palerme. Contemporaine des dernières années du théâtre de Charenton créé par Sade, La Casa dei Matti a été fondée en 1813 sur l'ordre de Joachim Murat, prince de Naples de 1808 à 1813 et beau-frère de Napoléon : il entend moderniser les institutions du royaume de Naples en transposant le modèle français. On y pratique le théâtre, sous forme de comédies deux fois par semaine ou de théâtre de marionnettes, la musique et les bals. Les malades tiennent les rôles, jouent au billard, dansent la tarentelle. Les voyageurs européens, souvent médecins eux-mêmes, qui ont pu assister à ces spectacles sont convaincus de leur vertu thérapeutique : ils le rapportent dans des publications médicales notamment dans les années $1830^{14}$. Cependant, c'est au caractère national italien, et surtout aux stéréotypes associés aux Napolitains (goût « naturel » pour la musique, la danse, le théâtre), que l'on attribue le plus souvent la réussite de ces moyens thérapeutiques novateurs.

L'expérience théâtrale menée par le marquis de Sade à l'hôpital de Charenton, antérieure, s'inscrit certes dans la réflexion des premiers aliénistes inventeurs du « traitement moral ». Mais elle a une autre portée, politique, au moment du tournant autoritaire de l'Empire et de la nécessité « impérieuse » ou impériale d'en

12 Ibidem, pp. 233-236. Mais du jour où l'ouvrier fut informé que la sentence n'était qu'une plaisanterie, il retomba dans son « ancien délire».

13 C. Raz, op. cit.

14 Voir par exemple, cités par C. Raz, op. cit. : G. Z. (anonyme), «Theater im königlichen Hause der Narren in Aversa », Allgemeine musikalische Zeitung 29, 1823 ; A. Brierre de Boismont, « Des établissements d'aliénés en Italie », Journal complémentaire des sciences médicales 13, Paris 1832 ; Anonyme, "Review of Account of the Present State of Medicine in Italy, by Fr.W. Oppenheim », Edinburgh Medical and Surgical Journal, vol. 25.149, 1825 ; J. Bell, Observations on Italy, vol. II, Fibreno, Naples 1835. 
finir avec l'Ancien Régime et ses figures dévoyées, dont Sade est une des plus encombrantes.

\section{LE THÉÂTRE DE CHARENTON (1805-1813)}

L'épisode, pour singulier qu'il soit, a été largement étudié. Pourtant de nombreuses questions demeurent, en particulier sur la participation effective des aliénés dans les spectacles conçus et mis en scène par le marquis de Sade. En 1803, Sade, qui aura passé 27 ans emprisonné (Bastille, Vincennes, Charenton), est à nouveau incarcéré, après quelques années de liberté, à l'hospice de Charenton rendu à sa première destination : le traitement de la maladie mentale.

François Simonet de Coulmier dirige l'établissement, assisté du médecin-chef Gastaldy ${ }^{15}$. Dans son Précis sur la maison de santé de Charenton, demeuré inédit mais consultable aux Archives du Val-de-Marne, Coulmier rappelle leur collaboration et décrit le projet thérapeutique :

Nous cherchions ensemble les moyens de les [les aliénés] dissiper par des jeux innocents, les concerts, la danse, des comédies dont les rôles étaient remplis par des malades, ce qui excitait entre eux une véritable émulation, par le désir d'en faire autant, de recevoir les mêmes applaudissements que leurs compagnons d'infortune. [...] Ce traitement moral, approuvé par les personnes les plus respectables, par des étrangers qui sollicitaient des billets d'entrée pour être les témoins de l'influence des arts sur le moral comme sur le physique, était parvenu à établir une réputation à la maison de Charenton ${ }^{16}$.

Coulmier prône donc le traitement moral et se réclame de Pinel. On abandonne la diète, les saignées, les purgatifs, les antispasmodiques. Concrètement cependant, à côté d'innocentes pratiques (promenades, jardinage, soin aux chevaux...), certains moyens « thérapeutiques » (« bains de surprise », enfermement dans des mannequins d'osier) ne manquent pas de violence. Mais l'innovation de Coulmier, celle qui restera dans l'histoire car associée à Sade, c'est le théâtre. Et le théâtre, prétend-il, joué par les malades eux-mêmes.

Il ne fait aucun doute que la présence de Sade à Charenton, surtout comme metteur en scène, mais aussi auteur de divertissements, a contribué à la réputation de la maison de Charenton et en particulier des spectacles organisés entre 1805 et 1813 (date de leur interdiction) : la maison semble s'être « imposé[e] dans la géographie des théâtres parisiens ${ }^{17}$. Que sait-on de cette expérience théâtrale ? Les malades y tenaient-ils réellement des rôles?

15 Voir la description du traitement en vigueur à Charenton donnée par Charles-François Giraudy, médecin adjoint de l'hospice, dans la biographie de Maurice Lever, Donatien Alphonse François, marquis de Sade, Fayard, Paris 1991, pp. 596-597.

16 Ibidem, p. 607.

17 J.L. Chappey, «Le nain, le médecin et le divin marquis. Folie et Politique à Charenton entre le Directoire et l'Empire », Annales historiques de la Révolution française 374, octobre-décembre 2013, p. 65. 
Il faut ici faire la part entre les critiques virulentes dont le théâtre de Charenton a fait l'objet par ses détracteurs - notamment Hippolyte de Colins dans sa Notice sur Charenton et, à sa suite, Esquirol, qui a selon Maurice Lever purement et simplement recopié Colins ${ }^{18}$-, et les témoins réels de ces spectacles, autrement dit les spectateurs. Le long témoignage de Mademoiselle Flore, actrice venue à Charenton dans l'espoir d'y rencontrer Madame de Saint-Aubin, grande chanteuse de l'Opéra-Comique qui honorait de sa présence ces représentations prolongées en soupers fins, a certes été publié tardivement (1845) ; mais très précise est la description que Flore donne de la représentation à laquelle elle assista, somptueux spectacle appelé la Fête de l'amitié, donnée en l'honneur de Coulmier. Cette fête s'ouvrait par Le Dépit amoureux de Molière :

Tous les rôles n'étaient pas joués par des fous ; on avait soin de les associer à des personnes de bon sens et à quelques comédiens de profession, pour maintenir l'équilibre. [...]

Le rôle d'Eraste était rempli par un jeune homme d'une physionomie très intéressante ; il était devenu, à ce que l'on m'a dit, fou par amour, après avoir perdu, par un accident subit, une jeune personne qu'il devait épouser. [...] La folle qui représentait Lucile était une jolie petite blonde, vive, gracieuse, qui joua parfaitement ses scènes de dépit et de coquetterie. La petite scène de Mascarille fut jouée très gaiement par un autre fou, dont le nom a été célèbre dans les lettres et au théâtre [...]. La première pièce marcha sans encombre [...]. La seconde pièce ne fut pas aussi heureuse que la première. [...] On fut obligé de baisser la toile ${ }^{19}$.

Le « joli opéra comique » Les Deux petits Savoyards suivit la deuxième pièce. Selon Flore, Madame Quesnet, comédienne et maîtresse en titre de Sade, joua dans la première ; la troisième pièce, dans laquelle jouait une belle pensionnaire espagnole du nom d'Urbistondos, fut une vraie réussite.

Un autre témoignage est celui du vaudevilliste Armand de Rochefort qui assista le 6 octobre 1812 à la fête donnée en l'honneur de Coulmier :

On nous conduisit dans une vaste salle où l'on avait construit un théâtre très-suffisant pour la circonstance ; c'était Marivaux qui devait faire les honneurs de la soirée ; avec sa comédie des Fausses confidences ; une musique d'une exécution irréprochable, qui avait été apprise par des fous se fit entendre, puis enfin un rideau fut ouvert et la pièce commença [...]. Une dame $\mathrm{L}^{* * *}$ (je craindrais de la nommer car je suppose qu'il lui reste une famille), parut dans le rôle si admirablement joué par Mademoiselle Mars ; la folle débita avec une aisance, une sûreté de mémoire qui confondait d'étonnement tous les spectateurs ; le personnage de l'amoureux était moins bien tenu, mais cependant l'acteur ne se trompait pas en donnant les répliques, enfin tout se termina sans erreur au milieu des applaudissements, de la surprise et de l'étonnement ${ }^{20}$.

S'il apparaît à travers ces témoignages que les malades ont un rôle limité, mais réel, dans l'interprétation des pièces données à Charenton, on est contraint de

18 M. Lever, op. cit., p. 645.

19 Ibidem, pp. 615-616.

20 A. de Rochefort, Mémoires d'un vaudevilliste, Charlieu et Huillery, Paris 1863, pp. 238241. Cité dans "Spectateurs et acteurs à Charenton », [dans :] Petits et grands théâtres du marquis de Sade, présentés par A. Lebrun, Art Center, Paris 1989, pp. 87-88. La ponctuation est celle de l'auteur de la lettre. 
lire entre les lignes pour déterminer la nature des spectateurs. Tout porte à croire que les aliénés eux-mêmes y ont peu de place, et que la plupart des spectateurs sont issus d'une société choisie. C'est ce que laisse entendre Armand de Rochefort lorsqu'il souligne « la surprise et l'étonnement» du public. Tout porte à croire aussi que les effets thérapeutiques du théâtre de Charenton sont eux-mêmes peu spectaculaires, en dépit du plaidoyer pro domo que tenta Sade dans la lettre qu'il adressa à sa cousine Madame Bimard le 4 mai 1811. Chef-d'œuvre de dialogisme, dans lequel il répond aux critiques :

J'ai dirigé le spectacle de la maison où je suis, et ce spectacle était un foyer d'horreur - L'eût-on souffert, autorisé, fréquenté six ans s'il eût été tel ? Eût-il guéri près de cinquante malades s'il eût ressemblé à cela ? ${ }^{21}$

Le Journal inédit de Sade 22 (1807-1814), permet par ailleurs de mesurer la complexité des rapports entre Sade et Coulmier : leurs relations, selon l'interprétation de Marie-Emmanuelle Plagnol-Diéval ${ }^{23}$, sont celles, classiques dans le théâtre de société, d'auteur et commanditaire, ce qui place en partie Sade dans une position de dépendance, mais inscrit également l'expérience de Charenton dans une tradition théâtrale historiquement marquée, celle du théâtre de société. Sollicité par exemple en janvier 1808 par le directeur pour mettre en scène la fête organisée en son honneur, Sade est aussi régulièrement en butte à ses critiques, justifiées en partie par les inquiétudes éprouvées par Coulmier quant à la réputation de son pensionnaire. Ce dont on a aussi une idée assez juste, c'est le dispositif théâtral de Charenton, situé au-dessus du bâtiment des femmes :

Ce théâtre dispose de toutes les commodités techniques d'un véritable théâtre : plateau, coulisses, loges, fosse d'orchestre, parterre, loge pour le directeur et ses invités, gradins de chaque côté de celle-ci. La salle est vaste, plus de 200 places si l'on additionne les places réservées pour le personnel (36), les malades (60) et les places retenues (90). La répartition est la suivante : 30 personnes dans les loges du bas, 38 dans celles du haut, 24 sur les bancs des côtés, 36 sur des bancs, 20 sur des chaises, plus 60 autres $^{24}$.

L'article de Marie-Emmanuelle Plagnol-Diéval, extrêmement informé, est une mine pour connaître les conditions concrètes de ces spectacles, organisés une fois par mois, et à ceci près, susceptibles d'être, dit-elle, assimilés au théâtre de société : agencement de plusieurs pièces (la petite et la grande), répertoire constitué surtout de comédies, autres divertissements (bals, ballets, concerts, soupers).

${ }^{21}$ D.A.F. de Sade, Lettre à Madame Bimard, Charenton, 4 mai 1811 (M. Lever, op. cit., p. 640).

22 D.A.F. de Sade, Journal inédit, Gallimard, Folio essais, Paris 1970.

23 M.-E. Plagnol-Diéval, « Marge dans la marge ou le théâtre de Sade à Charenton », [dans :] Théâtres en liberté du XVIII au XX siècle. Genres nouveaux, scènes marginales ?, V. Ponzetto (dir.), coll. «Actes de colloques et journées d'étude » de la CÉRÉdI, n 19, 2017. URL : <http:// ceredi.labos.univ-rouen.fr/public/?theatres-en-liberte-du-xviiie-au.html $>$ [consulté le 27.02.2019].

24 M.-E. Plagnol-Diéval, op. cit., p. 4. 
Sade joue parfois, il tient par exemple le rôle-titre dans L'Impertinent ou le billet perdu de Desmahis en juillet 1805, il chante à l'occasion.

Que joue-t-on à Charenton? La plupart des pièces ${ }^{25}$ sont des reprises de succès du siècle (Les fausses Confidences où deux pensionnaires tiennent les rôles principaux, Les Folies amoureuses de Regnard, Le nouveau Doyen de Killerine de Louis-Sébastien Mercier...), ou plus rarement du siècle précédent (Le Dépit amoureux de Molière), des vaudevilles accompagnés de musique. Le marquis conçoit de bout en bout la Fête de l'amitié donnée en l'honneur de Coulmier, qui y apparait comme un bienfaiteur consolateur et guérisseur sous le nom de Meilcour (anagramme de Coulmier, mais aussi nom d'un personnage des Égarements $d u$ cour et de l'esprit de Crébillon).

La place des « fous »dans la troupe des acteurs de Charenton reste un des points majeurs d'achoppement, et ce dès l'origine ou presque de l'expérience. Le mémoire, daté du 6 juin 1812, écrit par Hippolyte de Colins, ancien officier de cavalerie, sur l'hospice de Charenton, est particulièrement critique :

C'est un mensonge que de dire que ce sont les fous qui jouent la comédie à Charenton. Les véritables acteurs sont : un ancien infirmier devenu en dernier lieu le secrétaire du Directeur ; un marchand de vin du village ; le maître clerc d'un huissier du voisinage ; des femmes ou des jeunes personnes qui demeurent dans la maison, sans être ni avoir jamais été malades, des danseurs et des danseuses de l'Opéra, et enfin, quand le besoin l'exige, des hommes que l'on va chercher au café Touchard, rendez-vous des acteurs sans emploi, et à qui on paye et leur temps et leurs peines ${ }^{26}$.

Colins rapporte également un épisode fameux, celui où le danseur Trénitz, pensionnaire convalescent ou du moins « tranquille», fit scandale : engagé à danser le menuet de la Reine, on l'habille en Prince et c'est sous ces habits somptueux qu'il danse devant un public conquis. Mais lorsqu'il s'agit de le défaire de son costume, Trénitz, furieux d'avoir été dupé, doit être maîtrisé par la force. Du malade tranquille qu'il était, il aurait sombré dans la démence et l'agitation. Le successeur de Pinel, Jean-Étienne Esquirol, directeur de l'hospice de Charenton à partir de 1824, mais plus confiant dans les vertus curatives de la musique, conclut ainsi son Mémoire sur la maison de Charenton : « Ce qui se passait à Charenton nous l'apprend assez; que de rechutes, que d'accès de fureur provoqués par les représentations théâtrales ! jamais on n'a montré les individus guéris par ce mode de traitement $»^{27}$.

Au-delà de la question de l'efficacité thérapeutique de ce théâtre dans lequel les aliénés eux-mêmes ${ }^{28}$ ont donc un rôle limité, il faut souligner à quel point

${ }^{25}$ Ibidem, pp. 6-7.

${ }^{26}$ H. de Colins, Notice sur l'Hospice de Charenton, [dans :] D.A.F. de Sade, Journal inédit, p. 121.

27 J.-É. Esquirol, Mémoire historique et statistique sur la Maison royale de Charenton, extrait des Annales d'Hygiène publique, t. XIII, $1^{\text {ère }}$ partie, Paul Renouard, Paris 1835, p. 47.

${ }^{28}$ Et ce, d'autant plus que l'acteur est souvent présenté au XVIII ${ }^{\mathrm{e}}$ siècle, inspiré par la réflexion médicale antique, comme un être instable. Voir V. de Santis, « Maladies d'acteur. Théorie du jeu théâtral et littérature médicale au XVIII ${ }^{\mathrm{e}}$ siècle », Revue italienne d'études françaises 4, 2014. 
l'hospice de Charenton fut un lieu politique, théâtre aussi bien de luttes de pouvoir tout à fait révélatrices des enjeux politiques de la période. On reprochera à Coulmier sa gestion absolutiste et son propre dévoiement moral. Après le remplacement de son médecin chef Gastaldy par Athanase Royer-Collard, Coulmier perd progressivement sa liberté d'action, et les spectacles sont interdits en 1813. L'ordre impérial aura finalement raison de ce qui sera présenté comme une rémanence de l'aristocratie libertine de l'Ancien Régime.

Aujourd'hui, deux types de lecture semblent s'opposer concernant ce singulier épisode. D'une part, une lecture que l'on peut dire " classique », volontiers littéraire, qui vise à inscrire le théâtre de Charenton dans l'histoire du théâtre de société (qui n'est pas nécessairement aristocratique, il faut le rappeler). Certains chercheurs présentent Coulmier comme une sorte de charlatan ${ }^{29}$. D'autre part, une lecture de type libertaire, qui semble davantage le fait d'historiens et de philosophes : elle met l'accent sur l'inventivité et le caractère novateur, absolument inédit, de l'expérience de Charenton. C'est en particulier la lecture d'Annie Lebrun, qui a produit des travaux essentiels sur Sade et été commissaire de plusieurs expositions consacrées au divin Marquis. Annie Lebrun récuse notamment l'opposition courante qui est faite entre deux versants de l'œuvre de Sade, celui moral et conventionnel de son théâtre, celui scandaleux et pornographique que l'histoire a retenu. La coexistence des deux scènes, "théâtre de divertissement " et « théâtre des passions », serait selon elle liée à l'enfance, " rencontre de l'innocence et de la monstruosité dans un même être $»^{30}$.

Force est pourtant de constater que l'esprit révolutionnaire ne souffle pas dans le théâtre de Sade, même si le théâtre patriotique n'est certainement pas, dans ses codes et son traitement, des plus innovateurs.

En réalité, il est difficile de ne pas lire cet épisode à la lumière de notre histoire politique.

\section{LECTURES CONTEMPORAINES DE L'EXPÉRIENCE DE CHARENTON}

C'est pourquoi je dirai pour finir quelques mots des réécritures contemporaines de l'expérience théâtrale de Charenton. La plus célèbre est, représentée pour la première fois en 1964, celle de Peter Weiss, La Persécution et l'assassinat de Jean-Paul Marat représentés par le groupe théâtral de l'hospice de Charenton sous la direction de Monsieur de Sade, connue sous le titre court Marat/Sade.

29 C'est la critique que Jean-Luc Chappey adresse à la lecture de Marcel Gauchet et Gladys Swain dans leurs ouvrages Dialogue avec l'insensé, Gallimard, Paris 1993, et La pratique de l'esprit humain, Gallimard, Paris 1980.

${ }^{30}$ A. Le Brun, Soudain un bloc d'abîme, Sade, Jean-Jacques Pauvert et Grasset, Paris 1986 ; Folio, Gallimard, Paris 2014 [réédition], p. 162. 
La pièce est fondée sur la rencontre imaginaire entre Jean-Paul Marat et le marquis de Sade, qui n'a aucune réalité historique sinon que Sade prononça l'éloge funèbre de Marat. Peter Weiss écrit :

Ce qui nous intéresse dans la confrontation de Sade et de Marat, c'est le conflit entre l'individualisme poussé jusqu'à l'extrême et l'idée de bouleversement politique et social. Sade lui aussi était convaincu de la nécessité de la Révolution et ses œuvres sont de bout en bout une attaque contre la classe régnante corrompue, cependant il recule devant les mesures de terreur prises par les nouveaux dirigeants et se trouve, tel le représentant moderne du tiers parti, assis entre deux chaises $^{31}$.

On y voit Coulmier, qui expose le traitement moral, lequel fonde le dispositif de la pièce :

Nous autres esprits modernes et éclairés

Sommes d'avis qu'aujourd'hui dans ces murs

Les internés ne soient plus tenus par la terreur

Mais trouvent à s'exprimer par l'art et la culture

Illustrant par ce fait les principes sacrés

Que nous avons naguère à tout jamais gravés

Dans le solennel décret des Droits de l'Homme

Cette pièce dirigée par Monsieur Alphonse de Sade

Se déroulera dans notre salle de baignade

Toutes ces installations modernes de traitement

Ne présentent pour nous aucun inconvénient

Au contraire elles sont un décor tout trouvé

Pour la dramaturgie de Monsieur de Sade

puisque notre spectacle sera celui

de Jean-Paul Marat et de son agonie

laquelle eut lieu dans sa baignoire comme chacun sait

sous l'œil vigilant de Charlotte Corday ${ }^{32}$.

«Vrai » Sade donc, « vrai » Coulmier, tous les autres acteurs étant des internés de Charenton : Marat est joué par un paranoïaque, Charlotte Corday par une « hypotonique » se comportant en somnambule harcelée par un « érotomane », Duperret. On y voit un Sade désabusé : "Ma vie ce sont mes phantasmes. La Révolution ne m'intéresse plus ». Marat le lui reproche : «L'effervescence des pensées n'a jamais fait brèche dans aucune muraille. Ce n'est pas avec ta plume que tu briseras l'ordre qui règne $»^{33}$. Après l'assassinat de Marat, à la fin de la pièce, tous chantent $^{34}$ :

Charenton Charenton

Napoléon Napoléon

La Nation la Nation

31 P. Weiss, « Notes sur l'arrière-plan historique de la pièce », [dans :] idem, La Persécution et l'assassinat de Jean-Paul Marat..., traduit de l'allemand par J. Baudrillard, Éditions du Seuil, Paris 1965, p. 149.

32 Ibidem, pp. 14-15.

33 Ibidem, p. 52.

${ }^{34}$ Ibidem, p. 143. 
Révolution Révolution

Copulation Copulation.

Cette pièce, qui fera l'objet d'une adaptation filmée par Peter Brook (1967) et qui est régulièrement mise en scène, a trouvé sans peine un écho dans le courant libertaire que Mai 68 incarnera en partie. Elle dit aussi la confiscation et l'échec de la Révolution. Si la valeur subversive de la pièce a bien fonctionné jusque dans les années 80 , surtout grâce à la mise en scène de Brook ou lorsqu'elle a été portée par de grands acteurs comme Daniel Emilfork, est-elle aujourd'hui si convaincante ? Sans doute faut-il rester attentif à ses récentes mises en scène. Mais s'il est une pièce (objet d'un film lui aussi) qui donne une lecture assez ridicule de l'épisode de Charenton, c'est Quills de Doug Wright (1996) : sans aucune référence à la pratique théâtrale de Sade à Charenton, elle représente un Sade réduit à écrire avec ses excréments et mis en pièces, littéralement, par le pouvoir incarné par le docteur Royer-Collard. Le Canadien Robert Lepage, qui a co-signé la mise en scène qu'on a pu voir au théâtre de la Colline à Paris ${ }^{35}$, tentait de réactiver la charge subversive de la folie-Sade. Osons dire que la pièce ne présente guère d'intérêt historique, sinon qu'elle donne corps aux fantasmes courants appliqués au personnage de Sade lorsque la scène post-68 s'en empare : le divin Marquis devient le porte-parole des aliénés - cette fois au sens politique du terme. Quills ne présente guère davantage d'intérêt esthétique, du moins dans la mise en scène de Robert Lepage et Jean-Pierre Cloutier, si ce n'est l'interprétation qu'en donne le phénomène Lepage lui-même. Le jeu est dans l'ensemble convenu, et le décor, sans profondeur, n'est qu'une abstraction, fantasmée là encore, de l'asile de Charenton.

\section{CONCLUSION}

Pour conclure, on rappellera avec Laure Murat que la Révolution a produit tout à la fois de nouvelles pathologies et les expériences thérapeutiques censées les traiter. Mais ce n'est pas à la Révolution que l'on doit l'invention du traitement moral ni l'usage du théâtre comme moyen thérapeutique. Chez Pinel, le théâtre n'est pas nécessairement un moyen concerté, la mise en scène apparaît plutôt dans certains récits de cas comme un stratagème circonstanciel. Cette pratique peut du moins être inscrite, non sans précaution, dans une histoire : celle qui conduit au psychodrame analytique, individuel ou de groupe, comme moyen thérapeutique de la névrose.

On peut d'autre part poser l'hypothèse que le traitement moral suppose un nouveau regard sur le sujet et un nouveau rapport à la représentation, celui-là même qui est au principe de la Révolution. L'expérience théâtrale de Charenton menée par le marquis de Sade semble quant à elle relever davantage de pratiques

35 En février 2018. 
issues du théâtre de société florissant sous l'Ancien Régime. Mais cette expérience, si équivoque soit-elle, a une charge subversive telle qu'elle a inspiré à son tour, à partir du $\mathrm{XX}^{\mathrm{e}}$ siècle, des expérimentations théâtrales dont l'effet a anticipé ou croisé les moments « révolutionnaires » français de notre époque : Marat/Sade au premier chef. La Révolution française fait régulièrement retour dans le théâtre français contemporain : il y a eu 1789 d'Ariane Mnouchkine en 1970, en phase avec Mai 68 ; plus récemment, Notre Terreur de Sylvain Creuzevault (2009) ou Ça ira de Joël Pommerat (2015). Serpent de mer dont les circonvolutions rencontrent ou épousent les mouvements de révolte contemporains ? À moins que ces spectacles - encore faut-il les distinguer et les inscrire précisément dans le moment où ils ont été montés - n'aient eux aussi une certaine fonction thérapeutique : réveiller les consciences, ou consoler les nostalgiques de la Révolution en la reléguant sur la scène. Tout dépend du point de vue.

\section{THEATRE AND THERAPY FOR MENTAL ILLNESS}

(FRANCE, 1790-1815)

\section{Summary}

The theatrical experience conducted by the Marquis de Sade (1803-1814) with the lunatics of the Charenton asylum is a known fact. This practice, which was considered scandalous though it attracted the "Tout-Paris", was supposedly part of the so-called "moral treatment" initiated by the alienist-physician Philippe Pinel, founder of modern psychiatry in France. The transition period from the French Revolution to the Empire and the Restoration was, indeed, a time of many transformations in the treatment of mental illness. In its relation to mental illness, theatre may serve as a yardstick for measuring power issues related to divergent conceptions of man and his freedom. But this moment of history, observed through the prism of the problematic of theatre/mental illness, also makes it possible to question our constantly changing approaches to the French Revolution.

Key words: theatre, mental illness, French Revolution. 